\title{
Imaging Band-edge Localization in a Silicon Coupled Microring Waveguide (0925133-PO)
}

\author{
SHAYAN MOOKHERJEA \\ Department of Electrical and Computer Engineering, University of California, San Diego, MC 0407 La \\ Jolla CA 92093-0407 USA \\ Email:smookher@ucsd.edu
}

Summary of a Project Outcomes report of research funded by the U.S. National Science Foundation under Project Number 0925133. The band-edge localization of light in a coupled resonator optical waveguide (CROW) consisting of silicon microring resonators is studied theoretically and experimentally.

\section{Introduction and Goals}

Light propagates in a coupled-resonator optical waveguide (CROW) by nearest-neighbor coupling [1]. Borrowing from solid-state physics, researchers used a tight-binding model in early studies of the dispersion relationship of CROWs which is substantially different from that of a conventional waveguide and can support slow light near the band edge [2]-[6]. The model was later reformulated in a matrix eigenvalue problem to study the modes and resonance frequencies of the coupled-resonator system [7]. Longer CROW devices consisting of more than two hundred passive silicon resonators have been realized [8]. Once experimental measurements on these longer CROWs became available, certain aspects of the theory were further developed and a more detailed computational method was developed [9], [10]. To study the band-edge modes in more detail, silicon microring CROWs with $p$-n junctions in the waveguide cross-section were designed and fabricated using a multi-project wafer (MPW) process on silicon-on-insulator (SOI) wafers. The refractive index, and therefore, the optical propagation characteristics of these CROWs can be tuned by electrical means. Figure 1 shows part of a silicon CROW consisting of 51 coupled silicon microrings.

(a)

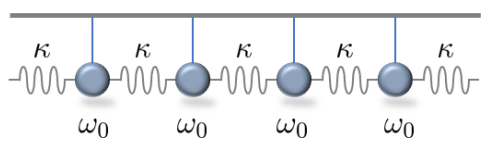

(b)

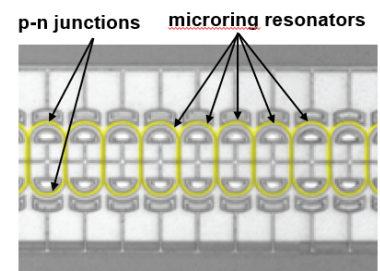

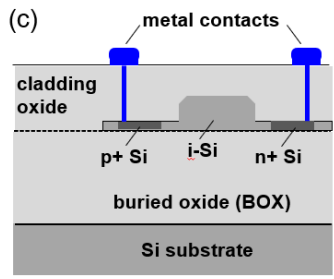

Figure 1 (a) An excitation can propagate in a chain of coupled resonators. (b) Realization of a coupled-resonator optical waveguide (CROW) using silicon microring resonators. (c) Cross section of the waveguide used to form the microring resonators. 


\section{Measurement and Analysis of the Modes}

Infrared imaging is a rapid and non-invasive way to study light propagation through CROW structures [11], [12]. Figure 2 shows a schematic of the experimental setup. Light transmission and imaging are performed simultaneously as the wavelength of the tunable laser is swept across a band of wavelengths.

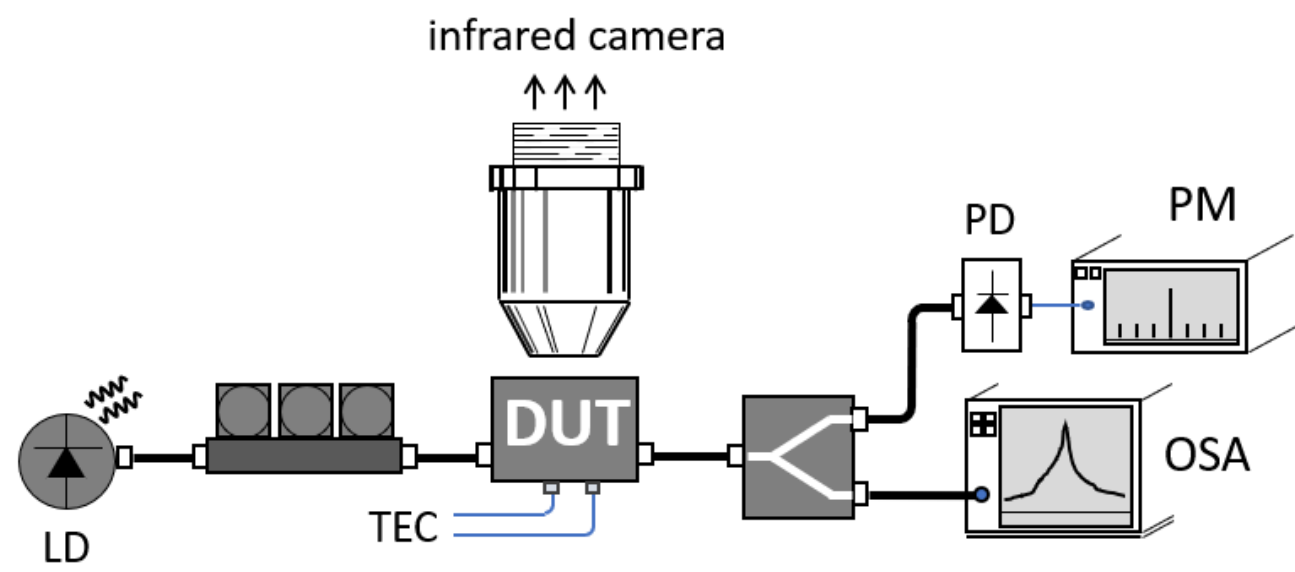

Figure 2 Measurement of light transmission through the device under test (DUT) using a tunablewavelength laser diode (LD), photodiode (PD), power meter (PM) and optical spectrum analyzer (OSA). The device is imaged using a microscope objective and an infrared camera.
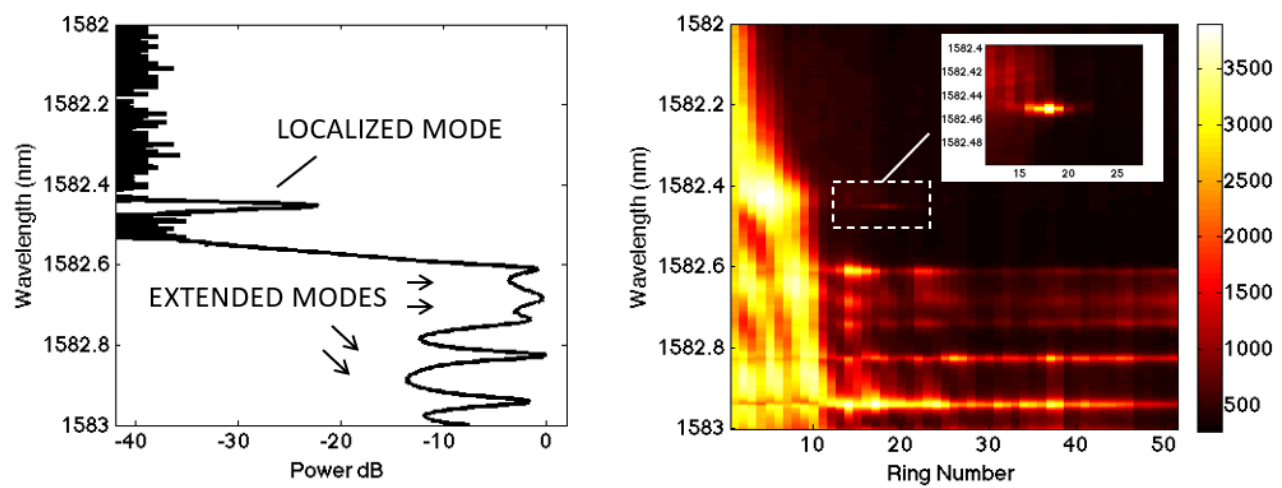

Figure 3 Wavelength-resolved transmission measurements and spatially-resolved infrared images of modes (after digital path-integration over each unit cell along the ring circumference). The inset shows a localized mode.

Figure 3 shows an example of a measurement in which the optical transmission and mode images were measured in this way. As the wavelength of the laser was stepped across the band edge and transmission band, the transmitted power was recorded, and is shown in Fig. 3(a). Simultaneously, infrared camera images of the CROW were acquired and processed offline using computer 
software. The camera readout over the circumference of each microring was digitally integrated, and the result is plotted in Fig. 3(b). A band-edge localized mode is seen, whose spatial extent is confined to a few rings. The mode is localized away from the edges of the structure and is exponentially confined [13]. This is shown more clearly in Fig. 4, which compares on the same colormap the captured images of an extended and a localized mode before the path integration is performed.
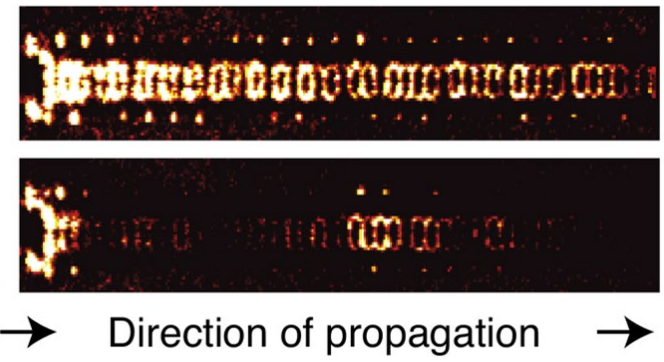

Figure 4 (top) Extended mode and (bottom) localized mode on the same colormap scale.

Figure 5 shows results of an ab-initio computation of modes in a coupledresonator chain with weak disorder. Similar to what was observed in the experiments, there can be localized modes at the band edge, which are spatially confined to a few resonators.
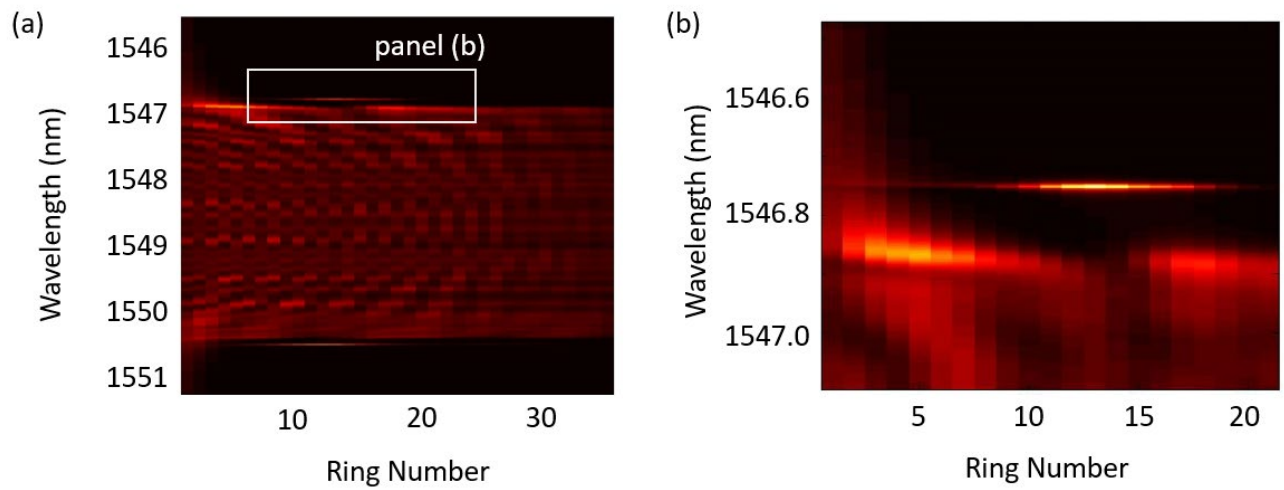

Figure 5 Calculations of the modes of a tight-binding resonator chain with disorder in the individual frequencies of the resonators. A localized mode near the band-edge is shown in more detail in panel (b).

\section{Design Alternatives and Subsequent Extensions.}

Localized modes are narrow-band excitations of trapped light. Light is localized in a small region without requiring lithographic patterning (other than in forming the $\mathrm{CROW}$ itself). The increased optical intensity relative to the 
propagating modes of a CROW can enhance the efficiency of light-matter interactions and nonlinear optics.

The bandpass transmission of CROWs is also useful, as an optical filter [14] or dispersion compensation devices [15] or a as a novel laser [16]. The velocity of propagation in the middle of the CROW band is slower than in a conventional waveguide. CROWs are useful for four-wave mixing and wavelength conversion [17]. Using spontaneous four-wave mixing, CROWs can also be used for generating entangled photon-pairs [18]-[20], although single microring resonators can also be used for this application [21].

Two aspects of multi-band operation are of special significance. Firstly, there can be dispersive variations in the band-to-band transmission of CROWs across a wide wavelength range [22], [23], which are a consequence of the high dispersion of the directional coupler [24], [25]. Secondly, if the device is used at higher optical power levels, the response of the directional coupler in silicon photonics can vary with optical power [26]. In contrast, wideband operation can be achieved even at elevated power levels using devices with moving-average (MA) rather than auto-regressive (AR) type transmission functions [27].

Diodes embedded in the waveguide cross-section of the silicon microring CROW shown in Figure 1 can be used to tune the (linear) optical propagation properties [13]. Nonlinear optical interactions can also be enhanced by using the diode, as was shown in later experiments using a single resonator [28]. Another fast but low-loss tuning mechanism would be achieved by incorporating a ferro-electric material such as lithium niobate, as has been shown with conventional Mach-Zehnder interferometers in a hybrid silicon platform, but not yet with CROWs [29].

These experiments and studies show that CROWs and their constituent microring resonators are sensitive to disorder. For precisely trimming resonators we have studied field-induced local oxidation of $\mathrm{Si}$ to $\mathrm{SiO}_{2}$ via a chemical reaction near an electrically-biased conducting atomic-force microscope (AFM) tip (used in contact mode) [30], [31]. This method can achieve a very high precision of trimming but is a slow process when using a conventional AFM scanner that is limited to $100 \mu \mathrm{m}$ scan regions. High-speed scanners are being developed which may be helpful for rapidly processing millimeter-length regions as required of CROWs [32].

\section{Open-Access Reporting Initiative}

PRAISE: This open-access document is provided in support of our PRAISE

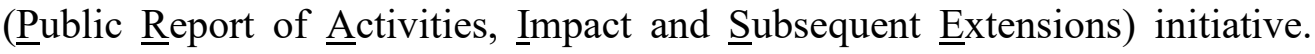
What is it? An open-access document shared with the public which describes 
the research outcomes of publicly-funded projects such as those funded by the U.S. NSF (National Science Foundation).

\section{References}

[1] A. Yariv, Y. Xu, R. K. Lee, and A. Scherer, "Coupled-resonator optical waveguide: a proposal and analysis," Opt. Lett., vol. 24, no. 11, pp. 711$713,1999$.

[2] S. Mookherjea, D. S. Cohen, and A. Yariv, "Nonlinear dispersion in a coupled-resonator optical waveguide," Opt. Lett., vol. 27, no. 11, p. 933, Jun. 2002, doi: 10.1364/OL.27.000933.

[3] S. Mookherjea and A. Yariv, "Optical pulse propagation and holographic storage in a coupled-resonator optical waveguide," Phys. Rev. E, vol. 64, no. 6, p. 066602, Nov. 2001, doi: 10.1103/PhysRevE.64.066602.

[4] S. Mookherjea and A. Yariv, "Pulse propagation in a coupled resonator optical waveguide to all orders of dispersion," Phys. Rev. E, vol. 65, no. 5, p. 056601, Apr. 2002, doi: 10.1103/PhysRevE.65.056601.

[5] S. Mookherjea and A. Yariv, "Kerr-stabilized super-resonant modes in coupled-resonator optical waveguides," Phys. Rev. E, vol. 66, no. 4, p. 046610, Oct. 2002, doi: 10.1103/PhysRevE.66.046610.

[6] S. Mookherjea and A. Yariv, "Optical pulse propagation in the tightbinding approximation," Opt. Express, vol. 9, no. 2, p. 91, Jul. 2001, doi: 10.1364/OE.9.000091.

[7] S. Mookherjea, "Spectral characteristics of coupled resonators," J. Opt. Soc. Am. B, vol. 23, no. 6, p. 1137, Jun. 2006, doi: 10.1364/JOSAB.23.001137.

[8] M. L. Cooper et al., "235-ring Coupled-Resonator Optical Waveguides," in Conference on Lasers and Electro-Optics 2010, San Jose, California, 2010, p. CTuHH3. doi: 10.1364/CLEO.2010.CTuHH3.

[9] S. Mookherjea and M. A. Schneider, "Avoiding bandwidth collapse in long chains of coupled optical microresonators," Opt. Lett., vol. 36, no. 23, p. 4557, Dec. 2011, doi: 10.1364/OL.36.004557.

[10] M. L. Cooper and S. Mookherjea, "Modeling of Multiband Transmission in Long Silicon Coupled-Resonator Optical Waveguides," IEEE Photon. Technol. Lett., vol. 23, no. 13, pp. 872-874, Jul. 2011, doi: 10.1109/LPT.2011.2141657.

[11] M. L. Cooper, G. Gupta, J. S. Park, M. A. Schneider, I. B. Divliansky, and S. Mookherjea, "Quantitative infrared imaging of silicon-on-insulator microring resonators," Opt. Lett., vol. 35, no. 5, p. 784, Mar. 2010, doi: 10.1364/OL.35.000784. 
[12] S. Mookherjea and H. R. Grant, "High dynamic range microscope infrared imaging of silicon nanophotonic devices," Opt. Lett., vol. 37, no. 22, p. 4705, Nov. 2012, doi: 10.1364/OL.37.004705.

[13] S. Mookherjea, J. R. Ong, X. Luo, and L. Guo-Qiang, "Electronic control of optical Anderson localization modes," Nature Nanotech, vol. 9, no. 5, pp. 365-371, May 2014, doi: 10.1038/nnano.2014.53.

[14] J. R. Ong, R. Kumar, and S. Mookherjea, "Silicon microring-based wavelength converter with integrated pump and signal suppression," Opt. Lett., vol. 39, no. 15, p. 4439, Aug. 2014, doi: 10.1364/OL.39.004439.

[15] S. Mookherjea, "Using gain to tune the dispersion relation of coupledresonator optical waveguides," IEEE Photon. Technol. Lett., vol. 18, no. 5, pp. 715-717, Mar. 2006, doi: 10.1109/LPT.2006.871144.

[16] S. Mookherjea, "Semiconductor coupled-resonator optical waveguide laser," Appl. Phys. Lett., vol. 84, no. 17, pp. 3265-3267, Apr. 2004, doi: 10.1063/1.1719278.

[17] J. R. Ong et al., "Low-power continuous-wave four-wave mixing in silicon coupled-resonator optical waveguides," Opt. Lett., vol. 36, no. 15, pp. 2964-2966, 2011.

[18] J. R. Ong and S. Mookherjea, "Quantum light generation on a silicon chip using waveguides and resonators," Opt. Express, vol. 21, no. 4, p. 5171, Feb. 2013, doi: 10.1364/OE.21.005171.

[19] R. Kumar, M. Savanier, J. R. Ong, and S. Mookherjea, "Entanglement measurement of a coupled silicon microring photon pair source," Opt. Express, vol. 23, no. 15, p. 19318, Jul. 2015, doi: 10.1364/OE.23.019318.

[20] R. Kumar, J. R. Ong, M. Savanier, and S. Mookherjea, "Controlling the spectrum of photons generated on a silicon nanophotonic chip," Nat Commun, vol. 5, no. 1, p. 5489, Dec. 2014, doi: 10.1038/ncomms6489.

[21] C. Ma, X. Wang, V. Anant, A. D. Beyer, M. D. Shaw, and S. Mookherjea, "Silicon photonic entangled photon-pair and heralded single photon generation with CAR $>12,000$ and $\mathrm{g}^{\wedge}(2)(0)<0006$, , Opt. Express, vol. 25, no. 26, p. 32995, Dec. 2017, doi: 10.1364/OE.25.032995.

[22] M. L. Cooper et al., "Waveguide dispersion effects in silicon-oninsulator coupled-resonator optical waveguides," Opt. Lett., vol. 35, no. 18, p. 3030, Sep. 2010, doi: 10.1364/OL.35.003030.

[23] S. Mookherjea, "Dispersion characteristics of coupled-resonator optical waveguides," Opt. Lett., vol. 30, no. 18, p. 2406, Sep. 2005, doi: 10.1364/OL.30.002406.

[24] R. Aguinaldo, Yiran Shen, and S. Mookherjea, "Large Dispersion of Silicon Directional Couplers Obtained via Wideband Microring Parametric Characterization," IEEE Photon. Technol. Lett., vol. 24, no. 14, pp. 1242-1244, Jul. 2012, doi: 10.1109/LPT.2012.2198639. 
[25] M. L. Cooper and S. Mookherjea, "Numerically-assisted coupledmode theory for silicon waveguide couplers and arrayed waveguides," Opt. Express, vol. 17, no. 3, p. 1583, Feb. 2009, doi: 10.1364/OE.17.001583.

[26] S. Mookherjea and M. A. Schneider, "The nonlinear microring adddrop filter," Opt. Express, vol. 16, no. 19, p. 15130, Sep. 2008, doi: 10.1364/OE.16.015130.

[27] R. Aguinaldo et al., "Wideband silicon-photonic thermo-optic switch in a wavelength-division multiplexed ring network," Opt. Express, vol. 22, no. 7, p. 8205, Apr. 2014, doi: 10.1364/OE.22.008205.

[28] M. Savanier, R. Kumar, and S. Mookherjea, "Optimizing photon-pair generation electronically using a $p-i-n$ diode incorporated in a silicon microring resonator," Appl. Phys. Lett., vol. 107, no. 13, p. 131101, Sep. 2015, doi: 10.1063/1.4932047.

[29] X. Wang, P. O. Weigel, J. Zhao, M. Ruesing, and S. Mookherjea, "Achieving beyond-100-GHz large-signal modulation bandwidth in hybrid silicon photonics Mach Zehnder modulators using thin film lithium niobate," APL Photonics, vol. 4, no. 9, p. 096101, Sep. 2019, doi: 10.1063/1.5115243.

[30] Y. Shen, I. B. Divliansky, D. N. Basov, and S. Mookherjea, "Perfect set-and-forget alignment of silicon photonic resonators and interferometers," in Optical Fiber Communication Conference/National Fiber Optic Engineers Conference 2011, Los Angeles, California, 2011, p. PDPC3. doi: 10.1364/OFC.2011.PDPC3.

[31] Y. Shen, I. B. Divliansky, D. N. Basov, and S. Mookherjea, "Electricfield-driven nano-oxidation trimming of silicon microrings and interferometers," Opt. Lett., vol. 36, no. 14, p. 2668, Jul. 2011, doi: 10.1364/OL.36.002668.

[32] T. Ando, "High-speed atomic force microscopy coming of age," Nanotechnology, vol. 23, no. 6, p. 062001, Feb. 2012, doi: 10.1088/0957$4484 / 23 / 6 / 062001$ 